SPECTRAL EVOLUTION OF HBV 475 (= V1329 CYGNI) IN THE ULTRAVIOLET

Ch. Kindl and H. Nussbaumer

Institute of Astronomy, ETH-Zentrum,

CH-8092 Zürich, Switzerland.

Figure 1 shows the low resolution IUE spectra of HBV 475 during 1979-81. Whereas the May 1981 spectrum looks very similar to that of June 1979, there is a clear difference in the August 1980 spectrum when the star was brighter. The strength of the NV multiplet at 1240 A surpasses those of the preceeding and succeeding years by at least a factor 4 . A first glance at the continuum and the strongest lines suggests a strong activity increase between June 1979 and August 1980, followed by a decrease to the 1979 level by May 1981. Closer inspection shows that neither CIII] $\lambda 1908$ nor NIII] $\lambda 1750$ followed that activity pattern, but rather decreased steadly in their fluxes. Thus in June 1979 CIII] $\lambda 1908$ showed the same flux as HeII $\lambda 1640$, whereas in May 1980 CIII] was weaker by a factor two. The OI $\lambda 1304$ and MgII $\lambda 2800$ multiplets show the same behaviour as HeII, CIV and NV.

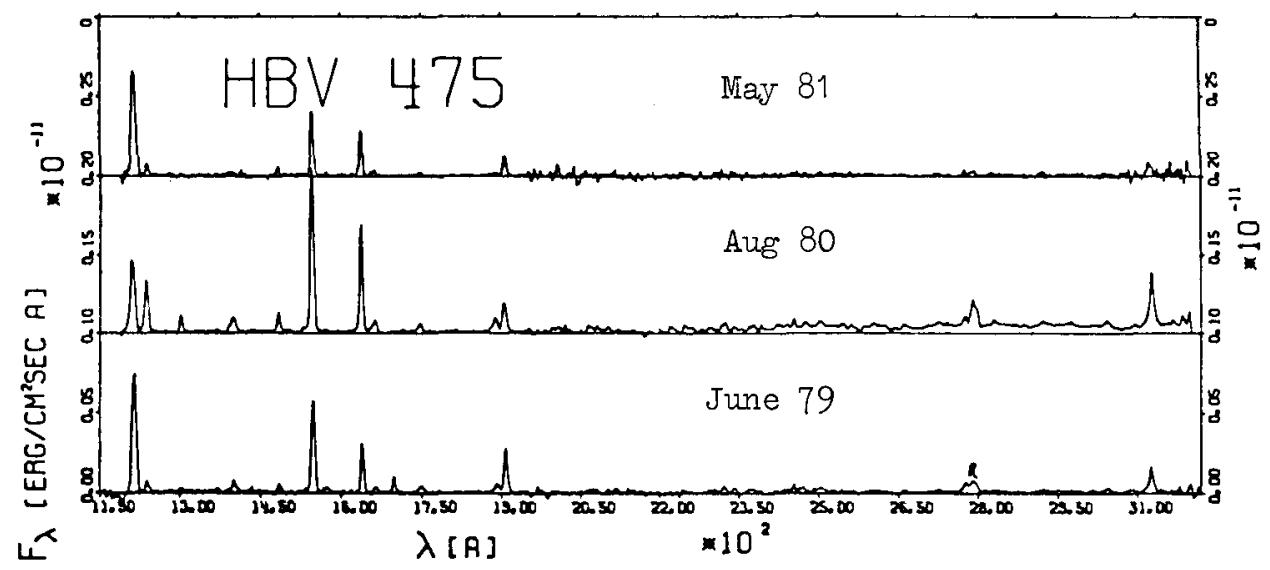

Figure 1. The low resolution ultraviolet spectrum of HBV 475.

M. Friedjung and R. Viotti (eds.), The Nature of Symbiotic Stars, 175-176.

Copyright $\odot 1982$ by D. Reidel Publishing Company. 


\section{DISCUSSION ON V1329 CYGNI}

Plavec: I think that the binary model of V1329 Cyg is fundamentally correct. The only problem is the large disparity of masses, $25 \mathrm{M}_{0}$ for the red star, $1 \mathrm{M}_{\Theta}$ for the hot star. Normally, components of binary systems tend to have much more equal masses. Naturally, here we have an old and evolved system, in which the hot star probably initially was the more massive one, and subsequently lost most of its mass. But we have an addi tional problem, namely the $25 \mathrm{M}_{\odot}$ for an $\mathrm{M}$ giant. All this is based on an unusually large mass function, which in turn is based on the radial velo city curve derived from the emission lines. Probably the lines do not re produce the velocity curve of the hot star. Both the large range and the eccentricity may be spurious, as they often are in interacting binaries.

Grygar: In our last solution (Grygar et al. 1979) we assumed a cir cular orbit and obtained $\mathrm{K}_{1}=(62 \pm 8) \mathrm{km} \mathrm{s}^{-1}$. Iijima et al. (1981) found the eccentricity $e=(0.17 \pm 0.09)$, but their radial velocity semi amplitude remained the same, i.e. $K_{1}=(63 \pm 6) \mathrm{km} \mathrm{s}^{-1}$.

Iijima: In my recurrent outburst model, the change of the radial velocity of the emission lines are explained with a hypothesis of moving ionization front. The 950 days is the period of the outbursts. The tempe rature of the exciting star changed owing to the outbursts. Therefore, the ionization front moves with the period of 950 days.

Slovak: Which are the magnitudes of V1329 Cyg at the time of the IUE observations?

Viotti: According the the AAVSO Circulars, V =12.95 on August 1980 near maximum, as compared to $V=13.4$ (June 1979) and $V=13.2$ (May 1981) was it was close to light minima. 